\title{
Analytical Sensitivity of Five SARS-CoV-2 Rapid Antigen Tests for Omicron Versus Delta Variant
}

\author{
Jean-Louis Bayart 1*, Jonathan Degosserie ${ }^{2,5}$, Julien Favresse ${ }^{3,4}$, Constant Gillot 4, Marie Didembourg 4 , Happy \\ Phanio Djokoto ${ }^{4}$,Valérie Verbelen ${ }^{1}$, Gatien Roussel 1, Celine Maschietto 2,5, François Mullier ${ }^{2,5}$, Jean-Michel \\ Dogné ${ }^{4}$ and Jonathan Douxfils ${ }^{4,6}$
}

1 Department of Laboratory Medicine, Clinique Saint-Pierre, 1340 Ottignies, Belgium; valerie.verbelen@cspo.be; gatien.roussel@cspo.be

2 COVID-19 federal testing platform and NGS surveillance consortium, CHU UCL Namur, 5530 Yvoir; Jonathan.degosserie@chuuclnamur.uclouvain.be; celine.maschietto@chuuclnamur.uclouvain.be

3 Department of Laboratory Medicine, Clinique Saint-Luc, 5004 Namur; j.favresse@labstluc.be

4 Department of Pharmacy, Namur Research Institute for Life Sciences, University of Namur, 5000 Namur, Belgium; constant.gillot@unamur.be; marie.didembourg@unamur.be; phanio.djokoto@unamur.be; jeanmichel.dogne@unamur.be; jonathan.douxfils@unamur.be;

5 Department of Laboratory Medicine, CHU UCL Namur, 5530 Yvoir; francois.mullier@chuuclnamur.uclouvain.be

6 Qualiblood S.A., 5000 Namur, Belgium

* Correspondence: jean-louis.bayart@cspo.be

\begin{abstract}
Rapid antigen detection tests (RAD) are commonly used for the diagnosis of SARS-CoV2 infections. However, with the continuous emergence of new variants of concern (VOC) presenting various mutations potentially affecting the nucleocapsid protein, the analytical performances of these assays should be frequently reevaluated. One-hundred and twenty samples were selected and tested with both RT-qPCR and five commercial RAD commonly sold in Belgian pharmacies. Of these, direct whole genome sequencing identified the strains present in 116 samples, of which 70 were Delta and 46 were Omicron. Sensitivity across a wide range of Ct values (13.5 to 35.7; median $=21.3$ ) were comparable and ranged from $70.0 \%$ to $77.1 \%$ for Delta strains and from $69.6 \%$ to $78.3 \%$ for Omicron strains. When taking swabs with a low viral load $(\mathrm{Ct}>25)$, poor performances were observed for the Delta strains (20.0 to $40.0 \%$ ) and, even more so, for Omicron strains (0.0 to $23.1 \%$ ). Two devices failed to detect all samples $(n=13)$ containing Omicron strains with a low viral load. The poor performance observed with low viral loads is an important limitation of RAD, which is not sufficiently highlighted in the instruction for use of these devices.
\end{abstract}

Keywords: SARS-CoV-2; variant; Omicron; Delta; antigen; RT-qPCR

\section{Introduction}

SARS-CoV-2 rapid antigen detection tests (RAD) are today widely used in the ongoing COVID-19 pandemic [1]. Thanks to the short period of time between the test procedure and the availability of the result, they can lead to a faster isolation of the patient and therefore limit virus spreading [2]. In Europe, the list of authorized RAD and their current field of application can be found on the European Commission website, which stipulates the rules governing the authorization of these RAD on the European market [3]. While the analytical performance of these devices were initially reported according to these procedures, few of them have evaluated the impact of various variant of concern (VOC) on their claimed specifications [4,5]. Recently, the emergence of the Omicron variant was shortly followed by a release note from the Food Drug Administration (FDA) stating that RAD may have reduced sensitivity for the Omicron variant [6]. The European Commission took 
the same cautiousness but precises that "[...] concerns have been raised about rapid antigen devices that are solely targeting the spike protein (thus not combined with the nucleocapsid protein) [...]" [3]. This warning, along with reports of undetected Omicron infected patients, led to a controversy about the reliability of RAD in the Omicron wave [7]. Given the critical role that RAD play in early case detection and their place in the testing strategy in many European countries, we studied and compared the performances of five RAD commonly sold in Belgium against the Delta and the Omicron variants.

\section{Materials and Methods}

Sixty positive specimens were collected during the "Delta" wave (between December $1^{\text {st }}$ and December $5^{\text {th }}$ ) and 60 other samples were collected during the Omicron wave (between January $1^{\text {st }}$ and January $\left.6^{\text {th }}\right)$ at Clinique Saint-Pierre, Ottignies, Belgium. Nasopharyngeal swabs were collected using Vacuette Virus Stabilization tubes (Greiner Bio-One, Kremsmünster, Austria). Samples were thereafter heat inactivated and RT-qPCR performed using the Allplex ${ }^{\circledR}$ 2019-CoV assay (Seegene, Arrow Diagnostics, Seoul, South Korea). This method uses a volume of $300 \mu \mathrm{L}$ and identify SARS-CoV-2 RNA by targeting four viral genes (N,E and RdRP/S), therefore fulfilling internationally validated testing procedures [8]. Viral load was expressed as cycle threshold (Ct) and samples with $\mathrm{Ct}$ values $<37$ for all three gene targets were considered as reactive for the present study. Ct values of the E gene were used as a proxy of the viral load. Following RT-qPCR analysis, specimens were frozen once at $-20^{\circ} \mathrm{C}$ until further analysis.

Among the 120 collected samples, 46 were confirmed by whole-genome sequencing (WGS) as being Omicron variant while 70 were Delta variant. Four samples could not be identified due to insufficient material. Median age of the patients was 33 and 35 years old in the Delta and Omicron groups, respectively. Asymptomatic cases represented $24 \%$ ( $\mathrm{n}=$ $17)$ and $37 \%(n=16)$ of the Delta and the Omicron groups, respectively.

Whole-genome sequencing (WGS) was performed following the "midnight" method with the Oxford Nanopore Rapid Barcode library kit, as described elsewhere [9]. Following WGS, the sequences were aligned with complete SARS-CoV-2 genomes of different lineages through the NextClade online tool [10]. Subsequently, sequences were analyzed using the Phylogenetic Assignment of Named Global Outbreak Lineages (PANGOLIN) software.

Five RAD tests commonly sold in Belgian pharmacies were used : (i) the Clinitest ${ }^{\circledR}$ Rapid COVID-19 Antigen test (Siemens Healthineers, Buizingen, Belgium), (ii) the Newgene COVID-19 Antigen Detection Kit (New-gene Bioengineering, Hangzhou, China), (iii) the Boson Rapid SARS-CoV-2 Antigen Test Card (Xiamen Boson Biotech Co., Xiamen, China), (iv) the Flowflex COVID-19 Antigen Home Test (Acon Laboratories, San Diego, USA) and (v) the Sejoy SARS-CoV-2 Antigen Rapid Test Cassette (Hangzhou Sejoy Electronics \& Instrument Co., Zhejiang, China). Briefly, samples previously analyzed for RTqPCR were unthawed and the appropriate number of droplets were dispensed on each device according to the recommendations of the manufacturers. Samples were identified as positive as both the control line and test line were present, as objectivated by two blinded and independent operators. The results of the five RAD were read after the reaction times recommended by the various manufacturers.

Descriptive statistics were performed using GraphPad Prism ${ }^{\circledR}$ software (version 9.0.1, California, CA, USA) and MedCalc ${ }^{\circledR}$ software (version 14.8.1, Ostend, Belgium). Sensitivity was defined as the proportion of samples qualified as positive by the RAD initially categorized as positive by RT-qPCR. A student's t-test was performed to assess statistical difference between cycle thresholds between RAD positive and negative groups.

The study was in accordance with the Declaration of Helsinki and was approved by the ethical committee of the Clinique Saint-Pierre Ottignies (approval number: 2020006149-21). Written informed consent was obtained for each patient. 


\section{Results}

Median Ct values were 20.83 and 21.37 in the Omicron and the Delta group, respectively $(p=0.37)$. Asymptomatic patients presented lower viral loads than symptomatic patients, both in the Delta group (median Ct; 31.1 vs 19.8; $\mathrm{p}<0.0001$ ) and the Omicron group (median Ct; 27.3 vs 19.4; $\mathrm{p}<0.0001$ ).

Among the 70 samples identified as Delta, 67 could be analyzed through the PANGOLIN online tool and characterized as belonging to the following Delta sub-lineages: AY.109 $(n=1)$, AY.110 $(n=1)$, AY.112 $(n=1)$, AY.118 $(n=1)$, AY.122 $(n=6)$, AY. $123(n=$ 1), AY. $127(\mathrm{n}=8)$, AY.34.1 $(\mathrm{n}=1)$, AY.4 $(\mathrm{n}=11)$, AY. $4.2(\mathrm{n}=3)$, AY.4.2.3 $(\mathrm{n}=1)$, AY.42 (n $=1)$, AY.43 $(n=22)$, AY.44 $(n=1)$, AY.46 $(n=1)$, AY.46.6 $(n=1)$, AY.5 $(n=3), A Y .9 .2(n=$ 1), AY.92 $(n=1)$ and B1.617.2 $(n=1)$ while Omicron strains were composed only of the two following types: BA.1 $(n=40)$, BA1.1 $(n=5) .3$ Delta and 1 Omicron strains could not be further characterized due to technical limitations.

Considering the whole cohort, the sensitivity of the RAD varied from $70.0 \%$ (Flowflex) to $77.1 \%$ (New-gene and Boson) in the Delta group. Calculated sensitivities were similar in the Omicron group and varied from $69.6 \%$ (Clinitest) to $78.3 \%$ (Boson). Sensitivity among patients with higher viral loads (i.e; $\mathrm{Ct} \leq 25$ ) were also similar in the Omicron group and varied between $91.2 \%$ (Flowflex) and $97.1 \%$ (New-gene and Boson) versus 95.6\% (Clinitest, New-gene and Sejoy) to $97.8 \%$ (Boson and Flowflex) in the Delta group. Furthermore, sensitivities among samples with low viral loads $(\mathrm{Ct}>25 ; \mathrm{n}=25$ and $\mathrm{n}=13$ in the Delta and Omicron groups, respectively) were poor for both cohorts. However, while this latter varied from 20 to $40 \%$ for the Delta group, two devices missed all low viral load samples in the Omicron group (Clinitest and Flowflex). The highest sensitivity was only $23.1 \%$ (Boson), which was able to detect 3 out of 13 samples (Table 1, Figure 1). Median $\mathrm{Ct}$ values between positive and negative RAD results were systematically significative $(\mathrm{p}<0.0001)$ in both groups (Figure 1).

Taking only asymptomatic cases, the calculated sensitivies varied from $31.3 \%$ (Clinitest and Flowflex) to 50\% (Boson) and from 23.5\% (New-gene and Flowflex) to $41.2 \%$ (Boson) for the Omicron and Delta groups respectively (Table 1).

Table 1. Comparison of the sensitivity of 5 RAD between the Delta and Omicron variant.

\begin{tabular}{ccccccc}
\hline Population & Variant & Clinitest & New-gene & Boson & Flowflex & Sejoy \\
\hline Total cohort & Delta & $72.9 \%$ & $75.7 \%$ & $77.1 \%$ & $70.0 \%$ & $74.3 \%$ \\
\hline
\end{tabular}




\begin{tabular}{|c|c|c|c|c|c|c|}
\hline & $(n=70)$ & $(60.9-82.8)$ & $(64.0-85.2)$ & $(65.6-86.3)$ & $(57.9-80.4)$ & $(62.4-84.0)$ \\
\hline & Omicron & $69.6 \%$ & $73.9 \%$ & $78.3 \%$ & $67.4 \%$ & $73.9 \%$ \\
\hline & $(n=46)$ & $(54.3-82.3)$ & $(58.9-85.7)$ & $(63.6-89.1)$ & $(52.0-80.5)$ & $(58.9-85.7)$ \\
\hline \multirow{4}{*}{$C t \leq 25$} & Delta & $95.6 \%$ & $95.6 \%$ & $97.8 \%$ & $97.8 \%$ & $95.6 \%$ \\
\hline & $(n=45)$ & $(84.9-99.5)$ & $(84.9-99.5)$ & $(88.2-99.9)$ & $(88.2-99.9)$ & $(84.9-99.5)$ \\
\hline & Omicron & $94.1 \%$ & $97.1 \%$ & $97.1 \%$ & $91.2 \%$ & $97.1 \%$ \\
\hline & $(n=33)$ & $(80.3-99.3)$ & $(84.7-99.9)$ & $(84.7-99.9)$ & $(76.3-98.1)$ & $(84.7-99.9)$ \\
\hline \multirow{4}{*}{$C t>25$} & Delta & $32.0 \%$ & $40.0 \%$ & $40.0 \%$ & $20.0 \%$ & $36.0 \%$ \\
\hline & $(n=25)$ & $(15.0-53.5)$ & $(21.1-61.3)$ & $(21.1-61.3)$ & $(6.8-40.7)$ & $(18.0-57.5)$ \\
\hline & Omicron & $0.0 \%$ & $7.7 \%$ & $23.1 \%$ & $0.0 \%$ & $7.7 \%$ \\
\hline & $(n=13)$ & $(0.0-24.7)$ & $(0.2-36.0)$ & $(5.0-5.38)$ & $(0.0-24.7)$ & $(0.2-36.0)$ \\
\hline \multirow{4}{*}{ Asymptomatic } & Delta & $35.3 \%$ & $23.5 \%$ & $41.2 \%$ & $23.5 \%$ & $35.3 \%$ \\
\hline & $(n=17)$ & $(14.2-61.7)$ & $(6.8-49.9)$ & $(18.4-67-1)$ & $(6.8-49.9)$ & $(14.2-61.7)$ \\
\hline & Omicron & $31.3 \%$ & $37.5 \%$ & $50.0 \%$ & $31.3 \%$ & $43.8 \%$ \\
\hline & $(n=16)$ & $(11.0-58.7)$ & $(15.2-64.6)$ & $(24.7-75.4)$ & $(11.0-58.7)$ & $(19.8-70.1)$ \\
\hline \multirow{4}{*}{ Symptomatic } & Delta & $84.9 \%$ & $92.5 \%$ & $88.7 \%$ & $84.9 \%$ & $86.8 \%$ \\
\hline & $(n=53)$ & $(72.4-93.3)$ & $(81.8-97.9)$ & $(77.0-95.7)$ & $(72.4-93.3)$ & $(76.7-94.5)$ \\
\hline & Omicron & $90.0 \%$ & $93.3 \%$ & $93.3 \%$ & $86.7 \%$ & $90.0 \%$ \\
\hline & $(n=30)$ & $(73.5-97.9)$ & (77.9-99.2) & $(77.9-99.2)$ & $(69.3-96.2)$ & (73.5-97.9) \\
\hline
\end{tabular}

Figure 1. Graphical representation of positive and negative antigen results according to RT-qPCR Ct values. A significant difference in the $\mathrm{Ct}$ value was observed between the positive and negative tests for each RAD.

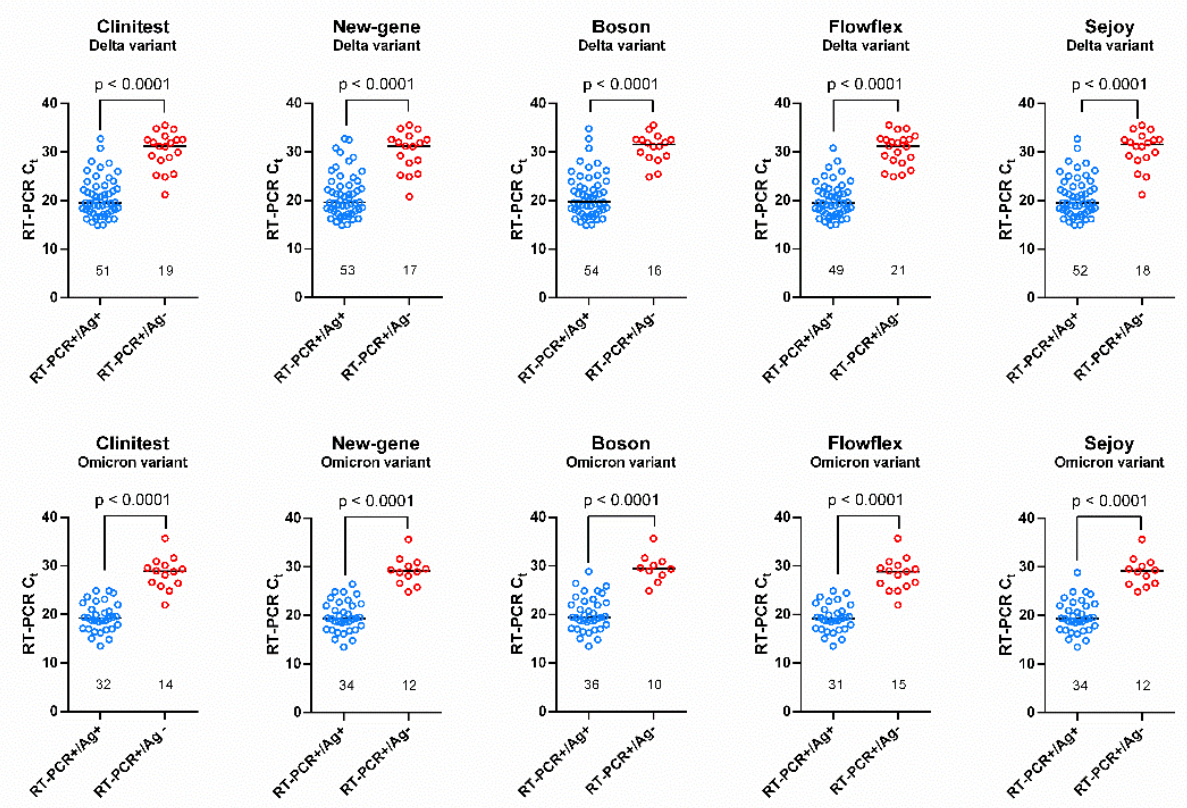

\section{Discussion}

The rapid spread of the Omicron variant in November 2021 was quickly followed by several cases reporting false negative $\operatorname{RAD}[7,11]$. Concomitantly, the FDA has publicly warned that RAD may be less sensitive for the Omicron variant, which led to some concerns about using this strategy to screen the population [6]. It is still unclear whether these findings are due to mutations accumulated within the nucleocapsid of the virus or 
whether this is due to variant-specific replication kinetics $[12,13]$. The latter explanation is further reinforced by several studies which reported higher viral loads in saliva compared to paired nasal swabs [7, 14]. In a high-risk occupational case cohort, Adamson et al. reported 30 cases were two RAD produced false-negative results on days 0 and 1 despite 28 out of these had potentially infectious viral load (i.e. $\mathrm{Ct}<29$, as considered in the setting of this study). The median time between the first positive PCR and positive RAD was 3 days while viral load in saliva peaked 1-2 days before the nose [7].

However, even if the Omicron viral load seems initially higher in saliva, RAD performed with cheek or throat swab alone seems not to be a good alternative due to a significantly reduced sensitivity compared to a classical nasal swab [15, 16]. Alternatively, nasal plus throat swabs increased the sensitivity from $64.5 \%$ to $88.7 \%$ compared to nasal swab only [15]. Moreover, some data emphasize the particular viral shedding of the Omicron variant with increased sensitivity when the RAD is performed several days following the first symptoms [7].

The sensitivities of the RAD for samples with Ct values $\leq 25$ reported in this study are quite comparable to previous study and is in line with the expected performance of these assays according to the clinical performance criteria for independent validation studies set up by the European HSC Technical Working Group on COVID-19 Diagnostic Tests [3, 4, 17-19]. Furthermore, in a systematic review and meta-analysis on the subject, Lee et al. reported a pooled sensitivity of $94 \%$. However, most of the published studies could not address the VOC in their analyses and whether distinct variants with potential mutations affecting the nucleocapsid protein could affect the sensitivity [20]. Reported sensitivities are largely variable across studies and some manufacturers do not meet current recommendations of the WHO which request a sensitivity $\geq 80 \%$ and a specificity $\geq$ $97 \%$ [20].

Reported sensitivities of RAD in samples with $\mathrm{Ct}>25$ are frequently low. The pooled sensitivity of $38 \%$ calculated by Lee $\mathrm{et} \mathrm{al}$. is in agreement with our values reported for the Delta variant, which vary from 20 to $40 \%$ [20]. Interestingly, in the samples containing Omicron strains, we found lower sensitivities, ranging from 0 to $23.1 \%$. These poor performances were also observed by Kanjilal et al. and by Landaverde et al. which observed a sensitivity below $5 \%$ to detect this category of samples $(\mathrm{Ct}>25)$ with the widely used BinaxNOW ${ }^{\circledast}$ RAD. These studies were however performed on a limited number of samples and did not compare several RAD [18, 21].

Samples with low viral load are commonly collected in asymptomatic patients. The five RAD evaluated in our study had low sensitivity (varying from $23.5 \%$ to $50 \%$ ) in this population, both in the Omicron and Delta groups. These results are in line with previous data $[22,23]$. These poor performances raise many questions about the testing strategy employed during the Omicron wave, where many asymptomatic patients performed RAD in their local pharmacies without confirmation with a RT-qPCR in case of negative result, which may certainly have increased the spread of the virus among the population.

Several studies have demonstrated the absence of variation in sensitivity for several RAD between major variants including Alpha, Beta, Gamma, Delta and the ancestral SARS-CoV-2 strain $[5,24,25]$. However, due to the plethora of devices on the market, continuous surveillance against the analytical performances of these RAD is mandatory. Salcedo et al. reported higher lowest limit of detection (LOD) for Delta (1,000 PFU/mL) compared to Alpha, Gamma (10 PFU/mL) and Omicron (100 PFU/mL). While extensive data concerning Omicron are still limited, initial experiments are reassuring and suggest that the current mutations P13L, Del31-33, R203K and G204R, observed in the sequence of the $\mathrm{N}$ gene for the Omicron BA.1 and BA1.1 sub-lineages, do not affect the sensitivity of commonly used RAD. On the other hand, Bekliz et al. found a large heterogenicity between rapid diagnostic tests for detecting Omicron, with a sensitivity varying from 22.2 to $88.9 \%$. Moreover, 4 out of their 7 RAD evaluated showed significantly lower sensitivity to detect Omicron when compared to Delta strains. These lower performances were observed both when assessed by infectious virus titers $(\mathrm{PFU} / \mathrm{mL})$ or by RNA copy numbers. 
While the design of this study was different from the present one, both observed a reduced sensitivity of RAD to detect Omicron in comparison to Delta strains [11].

Previous studies have identified specific mutations in the nucleocapsid affecting the sensitivity of RAD. Jian et al. identified in the Alpha variant the T135I mutation as a cause of false negative result with the Panbio COVID-19 RAD [26]. Furthermore, like reported by Bourassa et al., some mutations can affect only specific devices, as the D399N nucleocapsid mutation affected only the Quidel Sofia SARS Antigen FIA test but not five others RAD tested, including the extensively used BinaxNOW COVID-19 Ag card [27]. Finally, in their local study in the region of Veneto (Italy), Del Vecchio et al. reported that, when analyzing the discordant results between RT-qPCR and RAD, a relevant fraction of the circulating variants contained mutations A376T coupled to M241I in the nucleocapsid sequence. Viruses harboring this sequence were over-represented in the antigen-test-negative and PCR-positive samples and their prevalence increased over time in this region, which had extensively used antigen tests [28]. This study underlies how a mass utilization of RAD can create a selection pressure and enhance the spread of undetectable virus variants. In the present evaluation, WGS was not able to identify one or more specific mutations affecting the Omicron or Delta strains in the samples undetected by the various RAD. However, in light of our results and those of Bekliz et al., at equivalent viral loads, we cannot firmly exclude that RAD have lower sensitivity to detect the Omicron variant compared to other VOC, especially the Delta variant. This could be due to a lower affinity of monoclonal antibodies targeting the nucleocapsid in these devices. However, these antigenic targets are proprietary of the manufacturers and it is therefore unclear whether certain binding affinities could be affected by the specific mutations concerning the Omicron variant. At the time of writing these lines, BA.2 and BA. 3 are now the main Omicron sub-lineages circulating in Belgium. These latter are characterized by an additional mutation (S413R) which the impact on RAD performances is today unknown.

One limitation of this study if the sample modality, which was fully identical to specific manufacturers' recommendations, using a unique collection medium (Vacuette ${ }^{\circledR}$; containing Phosphate Buffered Saline solution). This methodology was however applied for both strains and for all tested RAD. It is anticipated that the comparison of their analytical performances remains valid.

\section{Conclusions}

We observed relatively comparable sensitivities between five commonly used RAD in Belgium. All devices presented good performance for detecting both Delta and Omicron variants in patients with high viral load $(\mathrm{Ct} \leq 25)$, with sensitivities varying from 91.2 to $97.8 \%$, in line with expectation of the European HSC Technical Working Group on COVID-19 Diagnostic Tests. RAD showed poor performance for patients with low viral loads $(\mathrm{Ct}>25)$ and in symptomatic patients Sensitivity among Omicron strains was lower than for Delta and some devices did not detect any Omicron samples presenting $\mathrm{Ct}>25$, though we could not identify a specific mutation pattern. The current testing strategy of not confirming negative RAD testing by RT-qPCR in asymptomatic cases certainly increased the spread of the pandemic these last months. This supports the need to limit the use of RAD for symptomatic cases.

Author Contributions: “Conceptualization, JL.B, J.F, J.DO and JM.DO.; methodology, JL.B, J.F, J.DO and JM.D; software, JL.B, C.M, C.G; validation, JL.B, J.F, J.DE and J.DO.; formal analysis, JL.B, J.DE, C.G, J.F; C.M, and J.DO; investigation, JL.B, J.DE J.F, M.D, HF.D and J.DO; resources, JL.B, J.DO and F.MU.; data curation, JL.B, C.G, C.M and J.DE.; writing-original draft preparation, JL.B; writing - review and editing, J.DE, J.FA and J.DO; visualization, JL.B; supervision, J.DO; project administration, J.DO and F.MU; funding acquisition, J.DO and JM.D All authors have read and agreed to the published version of the manuscript.

Funding: This research received no external funding 
Institutional Review Board Statement: Ethical review and approval were waived for this study since the hospital approves the usage of anonymized leftover patient samples collected for diagnostic purposes.

Informed Consent Statement: Informed consent was obtained from all subjects involved in the study.

Data Availability Statement: The data presented in this study are available on request from the corresponding author.

Acknowledgments: In this section, you can acknowledge any support given which is not covered by the author contribution or funding sections. This may include administrative and technical support, or donations in kind (e.g., materials used for experiments).

Conflicts of Interest: Among the authors, J.DO. is CEO and founder of QUALIblood s.a., a contract research organization manufacturing the DP-Filter, is co-inventor of the DP-Filter (patent application number: PCT/ET2019/052903) and reports personal fees from Daiichi-Sankyo, DOA-Sense, Gedeon Richter, Mithra Pharmaceuticals, Portola, Stago, Roche and Roche Diagnostics outside the submitted work. The other authors have no conflict of interest to disclose

\section{References}

1. Peeling, R. W.; Olliaro, P. L.; Boeras, D. I.; Fongwen, N., Scaling up COVID-19 rapid antigen tests: promises and challenges. The Lancet infectious diseases 2021, 21, (9), e290-e295.

2. Caruana, G.; Croxatto, A.; Kampouri, E.; Kritikos, A.; Opota, O.; Foerster, M.; Brouillet, R.; Senn, L.; Lienhard, R.; Egli, A., ImplemeNting SARS-CoV-2 Rapid antigen testing in the Emergency wArd of a Swiss univErsity hospital: the INCREASE study. Microorganisms 2021, 9, (4), 798.

3. https://ec.europa.eu/health/sites/default/files/preparedness_response/docs/covid-19 rat_common-list_en.pdf (16 february),

4. Favresse, J.; Gillot, C.; Oliveira, M.; Cadrobbi, J.; Elsen, M.; Eucher, C.; Laffineur, K.; Rosseels, C.; Van Eeckhoudt, S.; Nicolas, J.-B., Head-to-head comparison of rapid and automated antigen detection tests for the diagnosis of SARS-CoV-2 infection. Journal of clinical medicine 2021, 10, (2), 265.

5. Salcedo, N.; Nandu, N.; Boucau, J.; Herrera, B. B., Detection of SARS-CoV-2 Omicron, Delta, Alpha and Gamma variants using a rapid antigen test. medRxiv 2022.

6. Agency USFD. SARS-CoV-2 Viral Mutations: Impact on COVID-19 Tests. https://www.fda.gov/medicaldevices/coronavirus-covid-19-and-medical-devices/sars-cov-2-viral-mutations-impact-covid-19-

tests\#omicronvariantimpact (18 february),

7. Adamson, B. J.; Sikka, R.; Wyllie, A. L.; Premsrirut, P. K., Discordant SARS-CoV-2 PCR and Rapid Antigen Test Results When Infectious: A December 2021 Occupational Case Series. medRxiv 2022.

8. Bohn, M. K.; Mancini, N.; Loh, T. P.; Wang, C.-B.; Grimmler, M.; Gramegna, M.; Yuen, K.-Y.; Mueller, R.; Koch, D.; Sethi, S., IFCC interim guidelines on molecular testing of SARS-CoV-2 infection. Clinical Chemistry and Laboratory Medicine (CCLM) 2020, 58, (12), 1993-2000.

9. Freed, N. E.; Vlková, M.; Faisal, M. B.; Silander, O. K., Rapid and inexpensive whole-genome sequencing of SARS-CoV-2 using 1200 bp tiled amplicons and Oxford Nanopore Rapid Barcoding. Biology Methods and Protocols 2020, 5, (1), bpaa014.

10. https://clades.nextstrain.org/ (15 february),

11. Bekliz, M.; Adea, K.; Alvarez, C.; Essaidi-Laziosi, M.; Escadafal, C.; Kaiser, L.; Eckerle, I., Analytical sensitivity of seven SARS-CoV-2 antigen-detecting rapid tests for Omicron variant. medRxiv 2021.

12. Chan, M. C.; Hui, K. P.; Ho, J.; Cheung, M.-c.; Ng, K.-c.; Ching, R.; Lai, K.-1.; Kam, T.; Gu, H.; Sit, K.-Y., SARS-CoV-2 Omicron variant replication in human respiratory tract ex vivo. 2021. 
13. Peacock, T. P.; Brown, J. C.; Zhou, J.; Thakur, N.; Newman, J.; Kugathasan, R.; Sukhova, K.; Kaforou, M.; Bailey, D.; Barclay, W. S., The SARS-CoV-2 variant, Omicron, shows rapid replication in human primary nasal epithelial cultures and efficiently uses the endosomal route of entry. BioRxiv 2022, 2021.12. 31.474653.

14. Marais, G. J. K.; Hsiao, N.-y.; Iranzadeh, A.; Doolabh, D.; Enoch, A.; Chu, C. Y.; Williamson, C.; Brink, A.; Hardie, D. R., Saliva swabs are the preferred sample for Omicron detection. Medrxiv 2021.

15. Goodall, B. L.; LeBlanc, J. J.; Hatchette, T. F.; Barrett, L.; Patriquin, G., Investigating sensitivity of nasal or throat (ISNOT): A combination of both swabs increases sensitivity of SARS-CoV-2 rapid antigen tests. medRxiv 2022.

16. Schrom, J.; Marquez, C.; Pilarowski, G.; Wang, G.; Mitchell, A.; Puccinelli, R.; Black, D.; Rojas, S.; Riberio, S.; Martinez, J., Direct Comparison of SARS Co-V-2 Nasal RT-PCR and Rapid Antigen Test (BinaxNOW (TM)) at a Community Testing Site During an Omicron Surge. medRxiv 2022.

17. Krüttgen, A.; Cornelissen, C. G.; Dreher, M.; Hornef, M. W.; Imöhl, M.; Kleines, M., Comparison of the SARS-CoV-2 Rapid antigen test to the real star Sars-CoV-2 RT PCR kit. Journal of virological methods 2021, 288, 114024.

18. Kanjilal, S.; Chalise, S.; Shah, A. S.; Cheng, C.-A.; Senussi, Y.; Springer, M.; Walt, D. R., Analytic sensitivity of the Abbott BinaxNOW lateral flow immunochromatographic assay for the SARS-CoV-2 Omicron variant. medRxiv 2022.

19. de Michelena, P.; Torres, I.; Ramos-Garcia, A.; Gosalbes, V.; Ruiz, N.; Sanmartin, A.; Botija, P.; Puojois, S.; Huntley, D.; Albert, E., Real-life performance of a COVID-19 rapid antigen detection test targeting the SARS-CoV-2 nucleoprotein for diagnosis of COVID-19 due to the Omicron variant. medRxiv 2022.

20. Lee, J.; Song, J.-U.; Shim, S. R., Comparing the diagnostic accuracy of rapid antigen detection tests to real time polymerase chain reaction in the diagnosis of SARS-CoV-2 infection: A systematic review and meta-analysis. Journal of Clinical Virology 2021, 144, 104985.

21. Landaverde, L.; Turcinovic, J.; Doucette-Stamm, L.; Gonzales, K.; Platt, J.; Connor, J. H.; Klapperich, C. M., Comparison of BinaxNOW TM and SARS-CoV-2 qRT-PCR detection of the Omicron Variant from Matched Anterior Nares Swabs. medRxiv 2022.

Fernandez-Montero, A.; Argemi, J.; Rodríguez, J. A.; Ariño, A. H.; Moreno-Galarraga, L., Validation of a rapid antigen test as a screening tool for SARS-CoV-2 infection in asymptomatic populations. Sensitivity, specificity and predictive values. EClinicalMedicine 2021, 37, 100954.

23. Baro, B.; Rodo, P.; Ouchi, D.; Bordoy, A. E.; Amaro, E. N. S.; Salsench, S. V.; Molinos, S.; Alemany, A.; Ubals, M.; CorbachoMonné, M., Performance characteristics of five antigen-detecting rapid diagnostic test (Ag-RDT) for SARS-CoV-2 asymptomatic infection: a head-to-head benchmark comparison. Journal of Infection 2021, 82, (6), 269-275.

24. Weishampel, Z.; Young, J.; Fischl, M.; Fischer, R.; Owusu, I. O.; Riopelle, J.; Schulz, J.; Port, J. R.; Saturday, T.; van Doremalen, N., OraSure InteliSwab Rapid Antigen Test performance with the SARS-CoV-2 Variants of Concern Alpha, Beta, Gamma, Delta, and Omicron. medRxiv 2022.

25. Osterman, A.; Iglhaut, M.; Lehner, A.; Späth, P.; Stern, M.; Autenrieth, H.; Muenchhoff, M.; Graf, A.; Krebs, S.; Blum, H., Comparison of four commercial, automated antigen tests to detect SARS-CoV-2 variants of concern. Medical Microbiology and Immunology 2021, 210, (5), 263-275.

26. Jian, M.-J.; Chung, H.-Y.; Chang, C.-K.; Lin, J.-C.; Yeh, K.-M.; Chen, C.-W.; Lin, D.-Y.; Chang, F.-Y.; Hung, K.-S.; Perng, C.L., SARS-CoV-2 variants with T135I nucleocapsid mutations may affect antigen test performance. International Journal of Infectious Diseases 2022, 114, 112-114.

27. Bourassa, L.; Perchetti, G. A.; Phung, Q.; Lin, M. J.; Mills, M. G.; Roychoudhury, P.; Harmon, K. G.; Reed, J. C.; Greninger, A. L., A SARS-CoV-2 nucleocapsid variant that affects antigen test performance. Journal of Clinical Virology 2021, 141, 104900.

28. Del Vecchio, C.; Brancaccio, G.; Brazzale, A. R.; Lavezzo, E.; Onelia, F.; Franchin, E.; Manuto, L.; Bianca, F.; Cianci, V.; Cattelan, A., Emergence of N antigen SARS-CoV-2 genetic variants escaping detection of antigenic tests. medRxiv 2021. 
